\title{
Kinetics and Mechanism of the $\mathrm{NH}\left(\mathrm{X}^{3} \Sigma^{-}\right)+\mathrm{SO}\left(\mathrm{X}^{3} \Sigma^{-}\right)$Reaction, a Theoretical Approach
}

\author{
Nasim Hassani ${ }^{1}$, S. Hosein Mousavipour* 1,2 , Afshan Mohajeri ${ }^{1}$ \\ ${ }^{1}$ Department of Chemistry, College of Science, Shiraz University, Shiraz, Iran \\ ${ }^{2}$ Department of Chemistry, Faculty of Science, Sultan Qaboos University, Muscat, Sultanate of \\ Oman
}

\section{AUTHOR INFORMATION}

\section{Corresponding Author}

*E-mail: mousavipour@shirazu.ac.ir.

Phone: +98-7136137159. 
Scheme S1. The reaction mechanism proposed for the singlet surface of the NH + SO reaction.

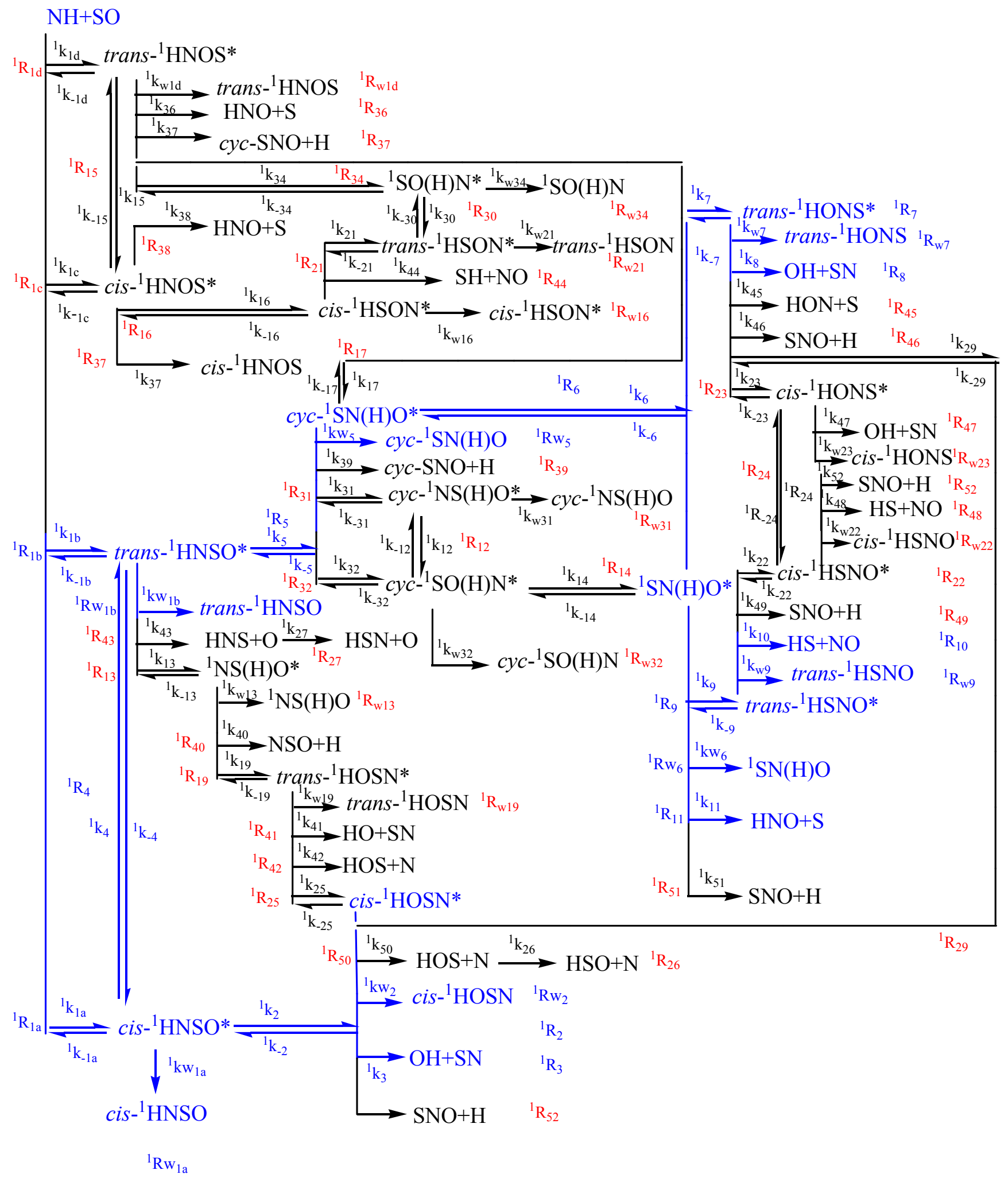

Scheme S2. The reaction mechanism proposed for the triplet surface of the NH + SO reaction. 


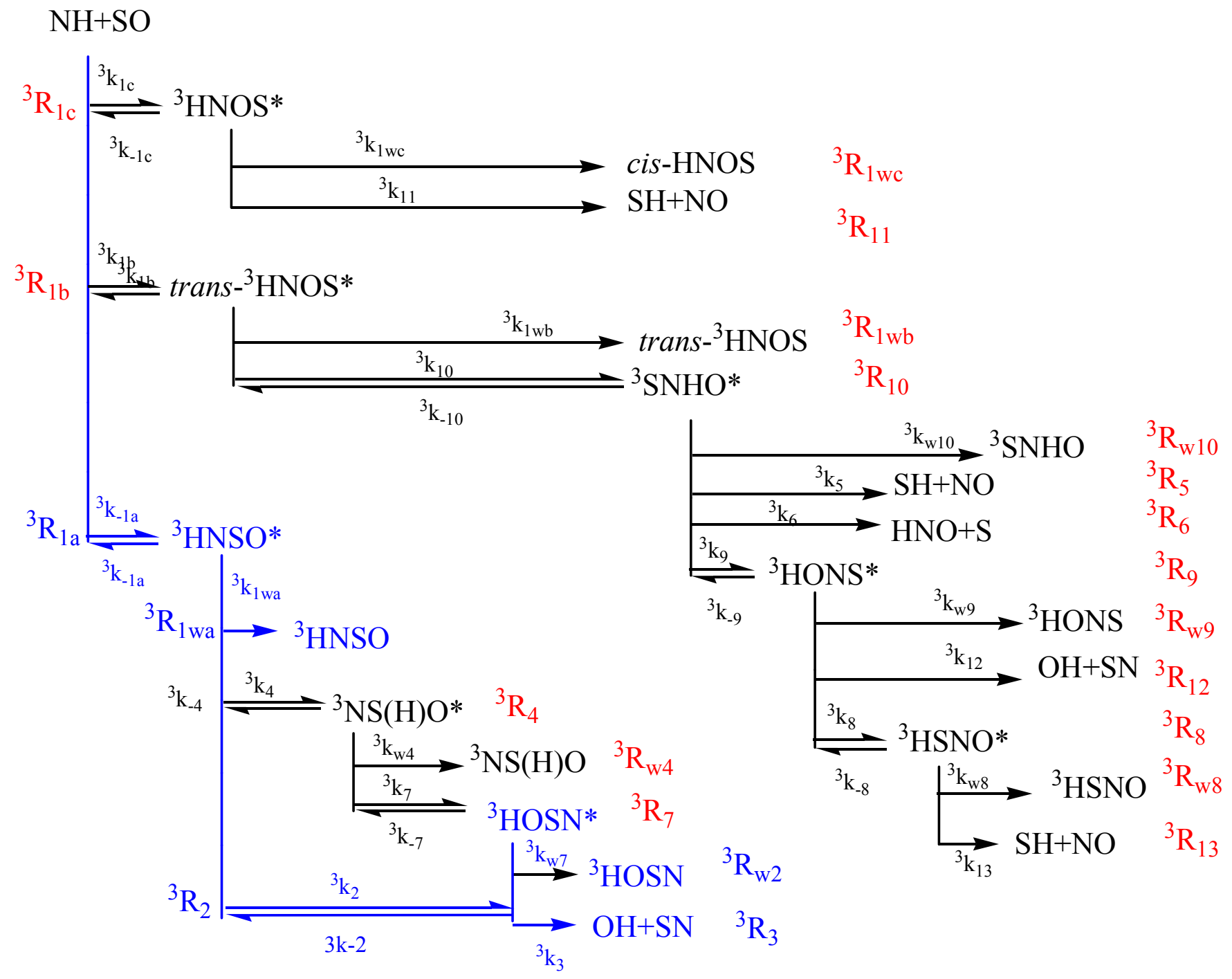

Table S1. Calculated enthalpies of formation $\left(\Delta \mathrm{H}_{\mathrm{f}}^{\circ}\right)(\mathrm{kJ} / \mathrm{mol})$ and percentage error $\left(|\Delta|^{\mathrm{a}}\right)$ for $\mathrm{OH}+\mathrm{SN}$ and $\mathrm{SH}+\mathrm{NO}$ species as major products in $\mathrm{NH}+\mathrm{SO}$ reaction at different levels of theory. 


\begin{tabular}{|c|c|c|c|c|}
\hline Method/Basis set & $\Delta \mathbf{H}_{\mathrm{f}}^{\circ}(0 \mathrm{~K})$ for $\mathrm{OH}+\mathrm{SN}$ & $|\Delta| \%$ & $\Delta \mathbf{H}_{\mathrm{f}}^{\circ}(0 \mathrm{~K})$ for $\mathrm{SH}+\mathrm{NO}$ & $|\Delta| \%$ \\
\hline CBS-QB3 & -53.4 & 17.3 & -134.0 & 0.8 \\
\hline CCSD(T)//B3LYP/CBSB7 & -44.8 & 1.6 & -133.7 & 0.6 \\
\hline G3MP2B3 & -43.1 & 5.3 & -127.7 & 3.9 \\
\hline PBE/6-311++G** & -48.0 & 5.4 & -155.4 & 16.9 \\
\hline PBE/Aug-cc-PVTZ & -36.8 & 19.2 & -134.6 & 1.3 \\
\hline M06-2X/6-311++G** & -46.9 & 3.0 & -156.6 & 17.8 \\
\hline M06-2X/Aug-cc-PVTZ & -34.6 & 24.0 & -132.6 & 0.2 \\
\hline BPW91/6-311++G** & -44.8 & 1.6 & -143.1 & 7.7 \\
\hline BPW91/Aug-cc-PVTZ & -33.7 & 26.0 & -129.4 & 2.6 \\
\hline MP2/6-311++G** & -36.0 & 20.9 & -141.5 & 6.5 \\
\hline MP2/Aug-cc-PVTZ & -32.4 & 28.8 & -124.5 & 6.3 \\
\hline Ref14 & -46.0 & 1.0 & -128.0 & 3.7 \\
\hline experiment & -45.5 & - & -132.9 & - \\
\hline
\end{tabular}

(S1) O'hare, P. A. G. Dissociation Energies, Enthalpies of Formation, Ionization Potentials, and Dipole Moments of NS and $\mathrm{NS}^{+}$. J. Chem. Phys. 1970, 52, 2992-2996.

(S2) Gurvich, L. V.; Veits, I. V.; Alcock, C. B. Thermodynamics Properties of Individual Substances. Volume 1Elements O, H/D, T/, F, Cl, Br, I, He, Ne, Ar, Kr, Xe, Rn, S, N, P, and Their Compounds. Part 1-Methods and Computation. Part 2-Tables. New York, Hemisphere Publishing Corp., 1989, P. Pt. 1, 577 P.; Pt. 2, 352 P. Translation. No Individual Items are abstracted in This Volume.

(S3) Ruscic, B.; Pinzon, R. E.; Morton, M. L.; Srinivasan, N. K.; Su, M. C.; Sutherland, J. W.; Michael, J. V. Active Thermochemical Tables: Accurate Enthalpy of Formation of Hydroperoxyl Radical, $\mathrm{HO}_{2}$. J. Phys. Chem. A 2006, $110,6592-6601$.

(S4) Chase, M.W.; Jr.; Davies, C.A.; Downey, J.R., Jr.; Frurip, D.J.; McDonald, R.A.; Syverud, A.N. JANAF Thermochemical Tables (Third Edition). J. Phys. Chem. Ref. Data,Suppl. 1, 1985, $14,1$.

Table S2. Calculated relative energies $\left(\mathrm{kJ} \mathrm{mol}^{-1}\right)$ of the stationary point observed on the singlet PES at different levels of theory. 


\begin{tabular}{|c|c|c|c|c|c|c|c|c|c|}
\hline \multirow[b]{2}{*}{$\begin{array}{l}\text { Stationary } \\
\text { Point }\end{array}$} & \multirow[t]{2}{*}{ G3MP2B3 } & \multicolumn{2}{|c|}{ BPW91 } & \multicolumn{2}{|c|}{ PBE } & \multicolumn{2}{|c|}{ M06-2X } & \multicolumn{2}{|c|}{ MP2 } \\
\hline & & $6-311++g * *$ & aug-cc-pVTZ & $6-311++\mathrm{g} * *$ & aug-cc-pVTZ & $6-311++\mathrm{g} * *$ & aug-cc-pVTZ & $6-311++\mathrm{g} * *$ & aug-cc-pVTZ \\
\hline $\mathrm{NH}+\mathrm{SO}$ & 0.0 & 0.0 & 0.0 & 0.0 & 0.0 & 0.0 & 0.0 & 0.0 & 0.0 \\
\hline $\mathrm{SH}+\mathrm{NO}$ & -127.7 & -143.1 & -129.4 & -155.4 & -134.6 & -156.6 & -132.6 & -141.5 & -124.5 \\
\hline $\mathrm{NS}+\mathrm{OH}$ & -43.1 & -44.88 & -33.7 & -48.0 & -36.8 & -46.9 & -34.6 & -36.0 & -32.4 \\
\hline$c i s-{ }^{1} \mathrm{HNOS}$ & -47.7 & -108.1 & -101.4 & -128.1 & -121.5 & -53.6 & -50.1 & -65.0 & -96.9 \\
\hline$c i s-1$ HNSO & -369.8 & -354.2 & -378.3 & -373.9 & -398.7 & -324.0 & -361.2 & -353.0 & -413.9 \\
\hline$c i s-{ }^{1}$ HONS & -201.6 & -251.8 & -243.2 & -273.0 & -264.8 & -231.0 & -223.1 & -255.5 & -243.4 \\
\hline$c i s-{ }^{1} H O S N$ & -304.4 & -299.6 & -309.4 & -318.5 & -329.2 & -276.8 & -297.9 & -278.3 & -323.9 \\
\hline$c i s-{ }^{1}$ HSNO & -232.8 & -298.0 & -282.4 & -317.6 & -301.9 & -275.8 & -244.1 & -246.0 & -254.6 \\
\hline$c i s-{ }^{1} H S O N$ & -123.5 & -182.7 & -161.3 & -200.8 & -178.4 & -92.9 & -70.5 & -145.1 & -146.4 \\
\hline trans-1HNOS & -43.2 & -101.4 & -93.2 & -120.9 & -112.9 & -49.0 & -43.4 & -70.1 & -96.5 \\
\hline trans-1 $-^{1} \mathrm{HNSO}$ & -358.5 & -336.5 & -365.8 & -356.4 & -386.3 & -307.4 & -349.7 & -336.5 & -413.9 \\
\hline trans-1HONS & -201.0 & -252.4 & -239.7 & -272.7 & -260.5 & -232.9 & -221.0 & -232.7 & --- \\
\hline trans-1'HOSN & -294.8 & -286.5 & -298.8 & -305.3 & -318.4 & -261.6 & -284.6 & -261.3 & -309.1 \\
\hline trans-1HSNO & -232.8 & -303.3 & -287.1 & -322.7 & -306.3 & -259.2 & -244.9 & -249.9 & -258.2 \\
\hline trans-1HSON & -124.4 & -185.6 & -163.7 & -203.7 & -180.7 & -92.9 & -70.5 & -144.0 & -144.1 \\
\hline${ }^{1} \mathrm{NS}(\mathrm{H}) \mathrm{O}$ & -164.3 & 131.0 & -159.0 & -151.9 & -180.1 & -81.4 & -123.0 & -106.6 & -178.7 \\
\hline${ }^{1} \mathrm{SN}(\mathrm{H}) \mathrm{O}$ & -202.8 & -278.5 & -262.6 & -300.1 & -283.9 & -236.9 & -224.0 & -249.9 & -262.9 \\
\hline${ }^{1} \mathrm{SO}(\mathrm{H}) \mathrm{N}$ & 243.6 & 156.6 & 173.7 & 136.8 & 154.1 & 205.0 & 217.5 & 286.1 & 271.2 \\
\hline$c y c-{ }^{1} \mathbf{N S}(\mathrm{H}) \mathrm{O}$ & 31.7 & - & 3.9 & - & -17.3 & 63.8 & 26.1 & 86.6 & 10.1 \\
\hline$c y c-{ }^{1} \mathrm{SN}(\mathrm{H}) \mathrm{O}$ & -89.9 & -116.7 & -129.3 & 136.5 & -150.3 & -113.8 & -128.5 & -90.9 & -144.0 \\
\hline$c y c-{ }^{1} \mathrm{SO}(\mathrm{H}) \mathrm{N}$ & 113.0 & - & 78.3 & - & 55.4 & 97.4 & 78.1 & 140.5 & 83.0 \\
\hline SNO+H & 36.1 & 35.1 & 20.5 & 52.3 & 37.3 & 27.2 & 39.7 & 0.6 & 5.4 \\
\hline $\mathrm{NSO}+\mathrm{H}$ & 86.4 & 49.2 & 26.7 & 34.4 & 11.5 & 105.3 & 70.8 & --- & 91.5 \\
\hline cyc-SNO+H & 189.1 & 161.7 & 154.1 & 145.8 & 137.2 & 191.9 & 182.4 & 222.0 & --- \\
\hline HSO+N & 339.8 & 400.6 & 219.2 & 402.0 & 220.4 & 379.4 & 373.8 & 437.5 & --- \\
\hline HOS+N & 364.1 & 406.7 & 237.5 & 407.6 & 238.4 & 364.8 & 372.8 & 407.3 & 406.5 \\
\hline $\mathrm{HON}+\mathrm{S}$ & 324.0 & 350.5 & 373.3 & 344.9 & 368.0 & 320.4 & 343.6 & 372.5 & 382.6 \\
\hline $\mathrm{HNO}+\mathrm{S}$ & 149.6 & 177.4 & 200.7 & 171.4 & 195.3 & 151.3 & 175.0 & 169.1 & 181.5 \\
\hline HNS+O & 323.0 & 416.2 & 432.0 & 415.2 & 430.9 & 363.1 & 377.0 & 393.8 & 399.6 \\
\hline $\mathrm{HSN}+\mathrm{O}$ & 394.6 & 501.6 & 511.1 & 501.5 & 510.7 & 463.8 & 469.1 & 516.1 & 515.2 \\
\hline${ }^{1}$ TS2 & -182.0 & -167.3 & -171.5 & -189.4 & -214.7 & -128.3 & -164.4 & -148.3 & -213.9 \\
\hline${ }^{1}$ TS4 & -313.7 & -290.6 & -313.8 & -311.1 & -334.9 & -263.6 & -299.1 & -283.6 & -374.2 \\
\hline${ }^{1}$ TS5 & -316.3 & -294.4 & -316.5 & -314.9 & -337.6 & -266.8 & -301.6 & -288.1 & -350.3 \\
\hline${ }^{1}$ TS6 & 30.1 & -112.4 & -99.4 & -77.4 & -71.0 & -67.5 & -72.7 & -80.9 & -71.5 \\
\hline${ }^{1}$ TS7 & -19.7 & -90.9 & -77.4 & -113.5 & -100.4 & -29.2 & -20.8 & -49.3 & -67.2 \\
\hline${ }^{1}$ TS9 & -40.5 & -112.6 & -99.4 & -135.9 & -122.7 & -223.9 & -209.2 & 194.7 & 196.3 \\
\hline${ }^{1}$ TS12 & 168.0 & 143.8 & 127.4 & 121.8 & 103.9 & 198.4 & 171.5 & 213.7 & 146.3 \\
\hline${ }^{1}$ TS13 & -69.0 & -36.5 & -80.9 & -58.8 & -90.5 & 28.1 & -14.2 & 0.4 & -78.1 \\
\hline${ }^{1}$ TS14 & -160.3 & -192.5 & -183.3 & -211.9 & -203.3 & -181.1 & -173.5 & -181.7 & -195.9 \\
\hline${ }^{1}$ TS15 & 84.3 & 16.5 & 23.8 & -1.5 & 5.5 & 60.6 & 65.4 & 74.9 & 44.1 \\
\hline${ }^{1}$ TS16 & 82.8 & 21.8 & 33.2 & 0.6 & 11.8 & 68.3 & 76.2 & 105.8 & 81.5 \\
\hline${ }^{1}$ TS17 & 145.0 & 18.2 & -6.6 & -28.9 & -70.5 & 51.1 & 52.6 & 46.0 & 44.0 \\
\hline${ }^{1}$ TS18 & 344.1 & - & 308.4 & 344.6 & 350.8 & 353.5 & 371.4 & - & --- \\
\hline${ }^{1}$ TS19 & -282.7 & -272.4 & -283.0 & -290.9 & -302.3 & -254.8 & -275.4 & -254.5 & -295.3 \\
\hline${ }^{1}$ TS20 & 347.5 & - & 308.4 & 301.5 & - & - & 384.0 & 437.9 & - \\
\hline${ }^{1}$ TS21 & -121.9 & -149.7 & -145.2 & -164.9 & -162.0 & -87.3 & -39.5 & -133.7 & -133.2 \\
\hline${ }^{1}$ TS22 & -124.4 & -256.0 & -240.4 & -275.1 & -258.9 & -224.0 & -209.0 & -220.0 & -227.1 \\
\hline${ }^{1}$ TS23 & -160.3 & -192.5 & -187.3 & -211.9 & -203.3 & -189.1 & -184.5 & -187.7 & -195.9 \\
\hline${ }^{1}$ TS24 & -113.0 & -174.1 & -165.1 & -196.9 & -188.5 & -123.2 & -115.6 & -122.8 & -145.5 \\
\hline${ }^{1}$ TS25 & -282.7 & -272.3 & -283.0 & -290.9 & -292.4 & -254.8 & -275.4 & -251.4 & -293.2 \\
\hline TS26 & 522.4 & - & 388.9 & 560.6 & 387.9 & 567.0 & 567.8 & 630.9 & - \\
\hline TS27 & 567.3 & - & 797.8 & 789.3 & 797.3 & 649.2 & 656.3 & 701.7 & - \\
\hline TS28 & 434.2 & - & 639.8 & - & - & 457.3 & 477.0 & 480.2 & - \\
\hline TS29 & -71.5 & -94.0 & -78.2 & -111.8 & -96.3 & -19.9 & -7.8 & -127.3 & -130.2 \\
\hline${ }^{1}$ TS30 & 217.9 & - & - & - & - & 263.0 & 273.4 & 338.3 & 364.1 \\
\hline${ }^{1}$ TS31 & 113.2 & 143.5 & 13.5 & -58.8 & -8.3 & -86.9 & - & 217.3 & - \\
\hline${ }^{1}$ TS32 & -61.4 & -192.5 & -78.2 & -211.9 & -203.4 & -19.9 & -7.7 & -123.8 & -124.3 \\
\hline${ }^{1}$ TS34 & 264.8 & 221.7 & 236.4 & 201.6 & 215.9 & 305.1 & 312.1 & 385.3 & 357.5 \\
\hline
\end{tabular}

Table S3. Calculated relative energies $\left(\mathrm{kJ} \mathrm{mol}^{-1}\right)$ of the stationary points on the triplet PES at different levels of theory. 


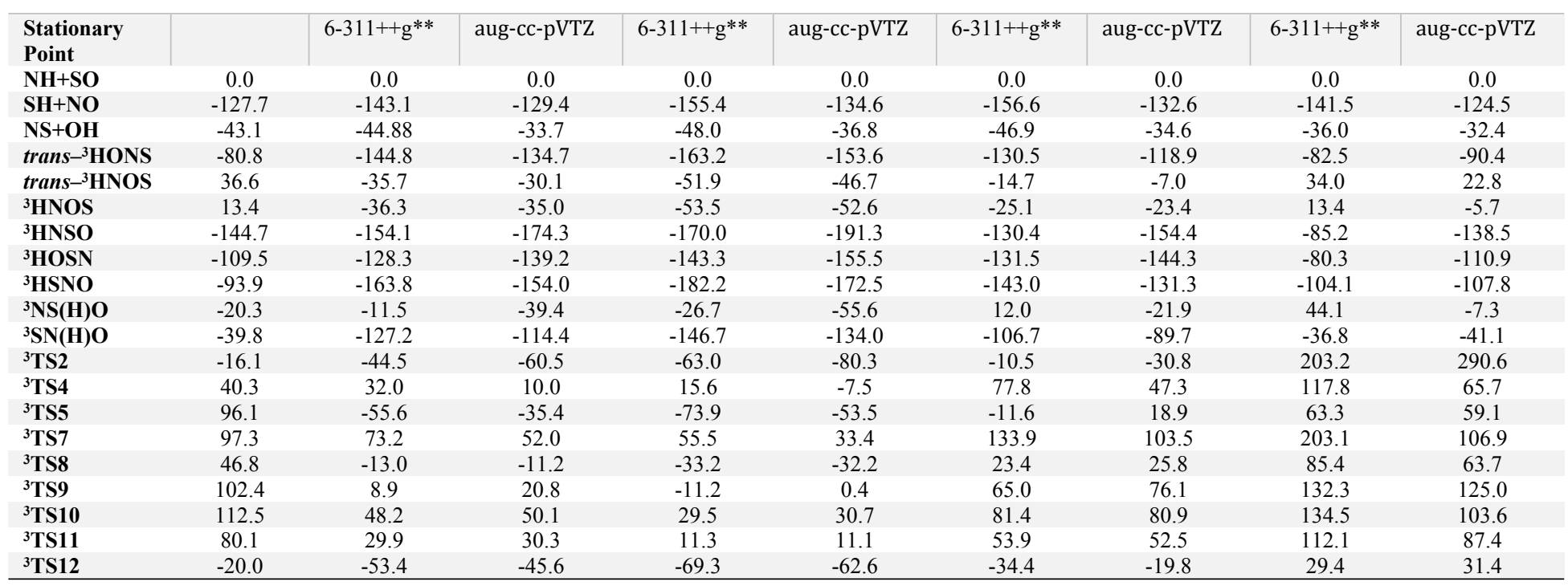

Table S4. Calculated vibrational term values and moments of inertia of the stationary points on the singlet surface by the CBS-QB3 method.

\begin{tabular}{|l|l|l|} 
Stationary Point & term values $\left(\mathbf{c m}^{-\mathbf{1}}\right)$ & $\mathbf{I}_{\mathbf{i}}(\mathbf{a . u})$. \\
NH & 3281.5 & $495.5,495.0$
\end{tabular}




\begin{tabular}{|c|c|c|}
\hline SO & 1121.4 & $21.1,21.1$ \\
\hline NS & 1241.5 & $23.1,23.1$ \\
\hline $\mathbf{O H}$ & 3704.7 & $560.5,560.5$ \\
\hline SH & 2667.4 & $284.8,284.8$ \\
\hline NO & 1988.4 & $51.3,51.3$ \\
\hline cis- ${ }^{1}$ HNOS & $449.3,624.5,819.5,1281.3,1524.4,3184.15$ & $81.7,6.6,6.1$ \\
\hline cis- ${ }^{1}$ HNSO & $447.0,793.2,690.9,1111.6,1254.2,3447.9$ & $48.3,9.8,8.1$ \\
\hline cis-11HONS & $512.9,735.1,929.5,1050.1,1419.2,3454.8$ & $68.9,7.1,6.4$ \\
\hline cis-1 $\mathrm{HOSN}$ & $363.6,449.1,635.3,1009.9,1361.4,321.9$ & $43.9,8.8,7.3$ \\
\hline cis-1'HSNO & $297.3,4128,508.1,857.2,1736.0,2648.1$ & $67.9,5.9,5.5$ \\
\hline cis-1 ${ }^{1}$ HSON & $185.0,232.1,372.0,583.1,1957.2,2659.5$ & $77.0,4.4,4.1$ \\
\hline trans- $-^{1}$ HNOS & $461.4,663.4,726.2,1213.3,1557.7,3311.1$ & $95.4,6.5,6.1$ \\
\hline trans $-{ }^{1}$ HNSO & $489.3,725.7,996.7,1065.4,1272.9,3458.0$ & $49.9,9.8,8.2$ \\
\hline trans $-{ }^{1} \mathrm{HONS}$ & $500.0,590.8,920.7,999.0,1417.7,3736.8$ & $75.9,6.8,6.3$ \\
\hline trans $-{ }^{1} \mathrm{HOSN}$ & $317.7,381.6,614.6,1003.6,1375.3,3745.0$ & $46.1,8.6,7.3$ \\
\hline trans $-{ }^{1}$ HSNO & $299.1,389.2,552.9,888.1,1756.2,2695.8$ & $71.3,5.8,5.4$ \\
\hline trans- $-^{1}$ HSON & $204.9,250.9,338.9,606.8,1961.6,2676.9$ & $80.2,4.3,4.1$ \\
\hline${ }^{1}$ NSHO & $475.4,526.0,1063.2,1092.3,1476.2,2453.5$ & $79.6,9.1,8.1$ \\
\hline${ }^{1}$ SNHO & $526.3,882.5,918.3,1485.7,1514.2,3251.4$ & $89.1,6.5,6.1$ \\
\hline${ }^{1}$ SOHN & $318.4,520.5,627.2,12054,1355.8,3279.4$ & $94.8,5.3,5.0$ \\
\hline$c y c-{ }^{1}$ NSHO & $420.6,619.3,829.0,945.2,1060.6,2318.4$ & $24.8,14.6,9.8$ \\
\hline cyc-1'SNHO & $595.1,674.8,966.0,1097.6,1363.5,3344.5$ & $28.8,12.3,8.9$ \\
\hline$c y c-{ }^{1}$ NOHS & $397.5,612.7,744.8,812.7,092.3,3694.6$ & $23.6,12.4,8.4$ \\
\hline SNO & $484.0,766.1,1653.2$ & $282.9,6.3,6.2$ \\
\hline NSO & $323.3,994.9,1186.7$ & $82.8,9.3,8.3$ \\
\hline$c y c-\mathrm{SNO}$ & $496.8,638.4,1114.8$ & $37.7,12.1,9.2$ \\
\hline HSO & $980.1,1079.9,2397.7$ & $297.3,19.9,18.7$ \\
\hline HOS & $794.8,1171.5,3758.3$ & $646.8,16.2,15.8$ \\
\hline HON & $1272.9,1482.2,3057.9$ & $654.9,39.7,37.4$ \\
\hline HNO & $1576.5,1673.9,2829.6$ & $556.3,42.9,39.8$ \\
\hline HNS & $1068.5,1241.5,3308.4$ & $587.2,19.4,18.8$ \\
\hline HSN & $1030.9,1167.5,2036.1$ & $299.3,22.1,20.6$ \\
\hline${ }^{1}$ TS2 & $1980.7 i, 615.7,971.8,1126.8,1229.7,1857.4$ & $30.9,12.3,8.8$ \\
\hline 1TS4 & $755.2 i, 393.8,502.7,1141.7,1328.2,3771.1$ & $56.5,9.5,8.1$ \\
\hline${ }^{1}$ TS5 & $963.8 i, 700.6,867.1,931.6,1165.6,3270.9$ & $17.4,16.3,8.6$ \\
\hline${ }^{1}$ TS6 & $989.4 i, 578.7,905.3,1185.7,1369.3,3235.4$ & $39.4,8.6,7.1$ \\
\hline${ }^{1}$ TS7 & $2087.6 i, 487.0,498.5,929.2,113.3,2592.7$ & $91.9,6.4,6.0$ \\
\hline 1TS9 & $2012.9 i, 48.0,424.0,801.1,1535.9,1853.1$ & $88.3,6.3,5.9$ \\
\hline${ }^{1}$ TS12 & $1578.4 i, 522.9,551.8,788.5,1152.7,1537.2$ & $27.8,12.6,8.9$ \\
\hline${ }^{1}$ TS13 & $1300.7 i, 320.4,451.5,1044.4,1319.9,2289.9$ & $65.6,9.1,8.2$ \\
\hline${ }^{1}$ TS14 & $642.1 i, 509.1,768.3,877.2,1074.4,3598.1$ & $25.8,11.53,8.2$ \\
\hline${ }^{1}$ TS15 & $554.8 i, 299.7,534.8,1287.6,1546.1,3102.2$ & $525.4,5.4,5.3$ \\
\hline${ }^{1}$ TS16 & $1961.9 i, 299.8,684.2,976.9,1469.9,1742.6$ & $55.1,6.8,6.1$ \\
\hline${ }^{1}$ TS17 & $733.7 i, 538.0,794.8,1126.7,1478.7,3186.8$ & $44.7,8.0,6.9$ \\
\hline TS18 & $433.1 i, 500.5,1279.5$ & $52.2,8.3,7.1$ \\
\hline${ }^{1}$ TS19 & $1646.1 i, 295.5,393.8,998.9,1411.0,1974.9$ & $71.9,8.9,7.9$ \\
\hline TS20 & $405.1 i, 126.7,1275.7$ & $26.9,8.6,6.5$ \\
\hline${ }^{1}$ TS21 & $198.8 i, 238.8,414.8,452.3,1965.4,2660.2$ & $72.3,4.3,4.1$ \\
\hline${ }^{1}$ TS22 & $403.9 i, 296.1,573.9,644.1,1852.9,2667.1$ & $65.0,5.3,5.0$ \\
\hline${ }^{1}$ TS23 & $727.4 i, 487.0,788.4,1039.0,1181.9,3749.2$ & $63.7,6.8,6.2$ \\
\hline${ }^{1}$ TS24 & $1727.9 i, 702.3,802.9,1014.7,1322.6,1731.4$ & $59.2,7.9,7.0$ \\
\hline${ }^{1}$ TS25 & $378.0 i, 386.9,610.0,895.2,1344.4,3764.8$ & $44.7,8.5,7.3$ \\
\hline TS26 & $1899.9 i, 759.8,2345.4$ & $417.5,16.7,16.1$ \\
\hline TS27 & $1909.1 i, 861.5,2448.3$ & $362.4,19.1,18.2$ \\
\hline TS28 & $2224.6 i, 1263.0,2847.5$ & $549.2,38.6,36.1$ \\
\hline 1TS29 & $509.7 i, 314.6,409.3,845.8,1361.0,3683.9$ & $26.9,7.9,6.1$ \\
\hline${ }^{1}$ TS30 & $1717.3 i, 228.8,314.9,692.8,1415.5,2146.8$ & $129.9,4.6,4.5$ \\
\hline${ }^{1}$ TS31 & $1016.0 i, 506.9,712.4,911.8,1117.9,2072.3$ & $29.4,12.2,9.0$ \\
\hline${ }^{1}$ TS32 & $1285.4 i, 496.6,643.9,775.8,1208.9,2475.9$ & $22.4,13.2,8.5$ \\
\hline${ }^{1}$ TS34 & $1954.7 i, 378.3,384.1,472.3,1078.4,2816.8$ & $99.1,5.6,5.3$ \\
\hline
\end{tabular}


Table S5. Calculated vibrational term values and moments of inertia of the stationary point on the triplet surface by the CBS-QB3 method.

\begin{tabular}{|c|c|c|}
\hline Stationary Point & term values $\left(\mathrm{cm}^{-1}\right)$ & $I_{i}$ (a.u.) \\
\hline$c i s-{ }^{3} \mathrm{HNOS}$ & $242.1,382.4,679.9,902.0,1438.6,3278.0$ & $56.3,6.9,6.2$ \\
\hline trans $-{ }^{3} \mathrm{HONS}$ & $254.4,357.8,787.5,1036.5,1375.7,3772.2$ & $97.9,6.1,5.8$ \\
\hline trans $-{ }^{3} \mathrm{HNOS}$ & $172.2,413.1,702.6,930.7,1442.2,3312.9$ & $62.9,6.7,6.1$ \\
\hline${ }^{3} \mathrm{HNSO}$ & $248.2,333.9,629.9,1015.6,1035.6,3343.8$ & $31.5,9.5,7.5$ \\
\hline${ }^{3} \mathrm{HOSN}$ & $272.7,323.7,663.5,817.0,1158.1,3759.8$ & $28.8,9.4,7.3$ \\
\hline${ }^{3} \mathrm{HSNO}$ & $211.3,383.3,488.2,906.2,1557.1,2587.6$ & $90.6,5.8,5.7$ \\
\hline${ }^{3} \mathrm{NS}(\mathrm{H}) \mathrm{O}$ & $278.9,550.0,796.8,986.7,1096.4,2242.4$ & $37.0,9.4,7.8$ \\
\hline${ }^{3} \mathrm{SN}(\mathrm{H}) \mathrm{O}$ & $298.6,644.5,772.5,1206.6,1402.5,3388.1$ & $56.3,6.9,6.2$ \\
\hline${ }^{3} \mathrm{TS} 2$ & $1808.1 i, 588.2,609.5,715.4,902.0,1970.4$ & $19.8,14.4,8.3$ \\
\hline${ }^{3}$ TS4 & $1760.8 i, 277.7,590.6,778.5,1112.6,1918.1$ & $37.9,8.8,7.4$ \\
\hline${ }^{3}$ TS5 & $1193.4 i, 211.3,366.5,1135.2,1633.9,2419.2$ & $71.2,4.2,4.0$ \\
\hline${ }^{3} \mathbf{T S} 7$ & $1382.0 i, 269.5,609.4,719.8,928.9,1862.9$ & $38.8,8.9,7.5$ \\
\hline${ }^{3}$ TS8 & $1923.5 i, 466.5,515.2,698.8,1030.3,1765.3$ & $41.4,8.4,7.0$ \\
\hline${ }^{3}$ TS9 & $1895.7 i, 389.9,550.6,753.8,964.6,2510.3$ & $112.4,5.7,5.5$ \\
\hline${ }^{3} \mathrm{TS} 10$ & $931.6 i, 188.3,497.2,1234.0,1275.3,3291.8$ & $35.5,8.8,7.1$ \\
\hline${ }^{3}$ TS11 & $1655.2 i, 512.2,537.4,670.0,1127.1,1813.6$ & $42.1,8.2,6.8$ \\
\hline${ }^{3}$ TS12 & $314.0 i, 83.8,324.5,718.8,1091.0,3727.1$ & $31.3,6.7,5.6$ \\
\hline
\end{tabular}

Table S6. Calculated relative energies ${ }^{a}\left(\mathrm{~kJ} \mathrm{~mol}^{-1}\right)$ of the OSN, SNO and $c y c-\mathrm{SNO}$ species at the $\mathrm{CASSCF} / \mathrm{cc}-\mathrm{pVXZ}(\mathrm{X}=\mathrm{D}, \mathrm{T})$ levels, at different active spaces.

\begin{tabular}{|c|c|c|c|c|c|c|c|c|c|c|c|}
\hline \multirow{3}{*}{$\begin{array}{l}\text { Stationary } \\
\text { Point }\end{array}$} & \multirow{3}{*}{ Ref14 } & \multicolumn{10}{|c|}{ Active spaces } \\
\hline & & \multicolumn{2}{|c|}{$(7,7)$} & \multicolumn{2}{|c|}{$(9,9)$} & \multicolumn{2}{|c|}{$(11,9)$} & \multicolumn{2}{|c|}{$(11,11)$} & \multicolumn{2}{|c|}{$(17,12)$} \\
\hline & & cc-pVDZ & cc-pVTZ & cc-pVDZ & cc-pVTZ & cc-PVDZ & cc-pVTZ & cc-PVDZ & cc-pVTZ & cc-PVDZ & cc-pVTZ \\
\hline OSN & 0.0 & 3.7 & 0.0 & 65.4 & 0.0 & 97.9 & 0.0 & 95.08 & 18.7 & 23.8 & 56.9 \\
\hline SNO & 7.11 & 0.0 & 61.4 & 0.0 & 48.6 & 0.0 & 36.3 & 0.0 & 0.0 & 0.0 & 0.0 \\
\hline cyc-SNO & 155.6 & 128.0 & 182.3 & 100.7 & 174.5 & 146.2 & 171.6 & 175.65 & 150.9 & 149.7 & 156.5 \\
\hline
\end{tabular}

Table S7. The structural parameters $(\mathrm{R}=$ bond lengths $=\AA$ ) of the OSN, SNO and $c y c-\mathrm{SNO}$ isomers calculated at the CASSCF/cc-pVXZ $(\mathrm{X}=\mathrm{D}, \mathrm{T})$ levels at three different active spaces.

\begin{tabular}{|c|c|c|c|c|c|}
\hline \multirow[b]{3}{*}{$\begin{array}{l}\text { Stationary } \\
\text { Point }\end{array}$} & \multirow{3}{*}{$\begin{array}{c}\text { structural } \\
\text { parameters }\end{array}$} & \multirow[b]{3}{*}{ Ref14 } & \multicolumn{3}{|c|}{ Active spaces } \\
\hline & & & $(7,7)$ & $(9,9)$ & $(11,9)$ \\
\hline & & & cc-pVTZ & cc-pVTZ & cc-pVTZ \\
\hline \multirow{3}{*}{ OSN } & $\mathrm{R}(\mathrm{SN})$ & 1.50 & 1.53 & 1.56 & 1.54 \\
\hline & $\mathrm{R}(\mathrm{SO})$ & 1.45 & 1.43 & 1.47 & 1.47 \\
\hline & $\mathrm{R}(\mathrm{NO})$ & 2.63 & 2.59 & 2.58 & 2.58 \\
\hline \multirow{3}{*}{ SNO } & $\mathrm{R}(\mathrm{SN})$ & 1.58 & 1.58 & 1.63 & 1.63 \\
\hline & $\mathrm{R}(\mathrm{SO})$ & 2.61 & 2.60 & 2.60 & 2.63 \\
\hline & $\mathrm{R}(\mathrm{NO})$ & 1.19 & 1.16 & 1.17 & 1.18 \\
\hline \multirow{3}{*}{$c y c$-SNO } & $\mathrm{R}(\mathrm{SN})$ & 1.70 & 1.73 & 1.67 & 1.77 \\
\hline & $\mathrm{R}(\mathrm{SO})$ & 1.74 & 1.78 & 1.74 & 1.78 \\
\hline & $\mathrm{R}(\mathrm{NO})$ & 1.37 & 1.33 & 1.40 & 1.36 \\
\hline
\end{tabular}

\section{The Parameters defined in the expression of the rate constants:}

$\boldsymbol{A 1}-\boldsymbol{A} 7$ parameters used in the rate constant calculations of Path1a on the singlet surface are defined as: 


$$
\begin{aligned}
& A 1=k_{-1 a}(E)+k_{4}(E)+w \\
& A 2=k_{5}(E)+k_{6}(E)+w \\
& A 3=\left(k_{-2}(E) k_{2}(E)\right) /(A 1 * A 2) \\
& A 4=1-A 3 \\
& A 5=A 1 * A 2 * A 4 \\
& A 6=k_{3}(E) * k_{2}(E) \\
& A 7=A 6 / A 5
\end{aligned}
$$

D1 -D32 parameters used in the rate constant calculations of Path1b on the singlet surface are defined as:

$$
\begin{aligned}
& D 1=k_{-4}(E)+k_{2}(E)+w \\
& D 2=k_{-2}(E)+k_{3}(E)+w \\
& D 3=\left(k_{-2}(E) * k_{2}(E)\right) / D 2 \\
& D 4=D 1-D 2 \\
& D 5=k_{-5}(E)+k_{6}(E)+w \\
& D 6=k_{-1 b}(E)+k_{5}(E)+k_{4}(E)+w \\
& D 7=\left(k_{-4}(E) * k_{4}(E)\right) / D 4 \\
& D 8=D 6_{-D} D 7 \\
& D 9=k_{5}(E) / D 8 \\
& D 10=\left(k_{-5}(E) * k_{5}(E)\right) / D 8 \\
& D 11=D 5_{-} D 10 \\
& D 12=\left(k_{-5}(E) * D 9\right) / D 11 \\
& D 13=k_{-6}(E) / D 11 \\
& D 14=k_{-7}(E)+k_{8}(E)+w \\
& D 15=k_{-9}(E)+k_{10}(E)+w \\
& D 16=k_{-6}(E)+k_{7}(E)+k_{11}(E)+k_{9}(E)+w \\
& D 17=D 9 / D 11 \\
& D 18=\left(k_{-6}(E) * k_{6}(E)\right) / D 11 \\
& D 19=\left(k_{-7}(E) * k_{7}(E)\right) / D 14 \\
& D 20=\left(k_{-9}(E) * k_{9}(E)\right) / D 15 \\
& D 10
\end{aligned}
$$




$$
\begin{aligned}
& D 21=D 16-D 18-D 19-D 20 \\
& D 22=D 17 / D 21 \\
& D 23=\left(k_{7}(E) * D 22\right) / D 14 \\
& D 24=\left(k_{9}(E) * D 22\right) / D 15 \\
& D 25=1+D 12 \\
& D 26=D 13 * D 22 \\
& D 27=(D 25+D 26) / D 8 \\
& D 28=k_{-6}(E) * D 22 \\
& D 29=(D 9+D 28) / D 11 \\
& D 30=k_{2}(E) * D 27 \\
& D 31=\left(k_{2}(E) * D 30\right) /(D 4 * D 2) \\
& D 32=D 30 / D 4
\end{aligned}
$$

$\boldsymbol{B} 1-\boldsymbol{B} 7$ parameters used in the rate constant calculations of Path1a on the triplet surface are defined as:

$$
\begin{aligned}
& B 1=k_{-1 a}(E)+k_{4}(E)+w \\
& B 2=k_{5}(E)+k_{6}(E)+w \\
& B 3=\left(k_{-2}(E) k_{2}(E)\right) /(B 1 * B 2) \\
& B 4=1-B 3 \\
& B 5=B 1 * B 2 * B 4 \\
& B 6=k_{3}(E) * k_{2}(E) \\
& B 7=B 6 / B 5
\end{aligned}
$$



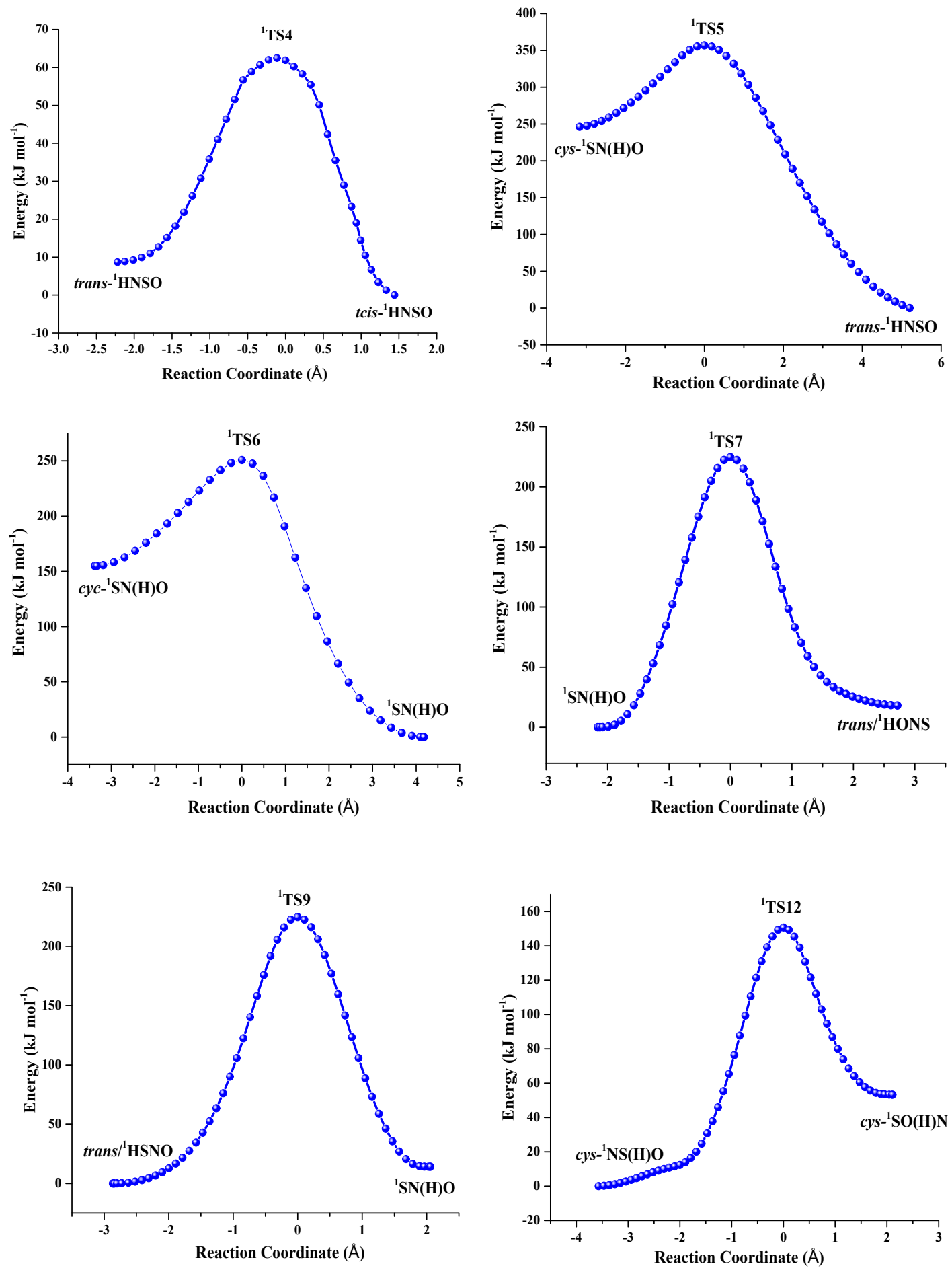

Figure S1. Calculated IRC for ${ }^{1} \mathrm{R}_{4},{ }^{1} \mathrm{R}_{5},{ }^{1} \mathrm{R}_{6},{ }^{1} \mathrm{R}_{7},{ }^{1} \mathrm{R}_{9}$, and ${ }^{1} \mathrm{R}_{12}$ reactions at $\mathrm{B} 3 \mathrm{LYP} / \mathrm{CBSB} 7$ level. 

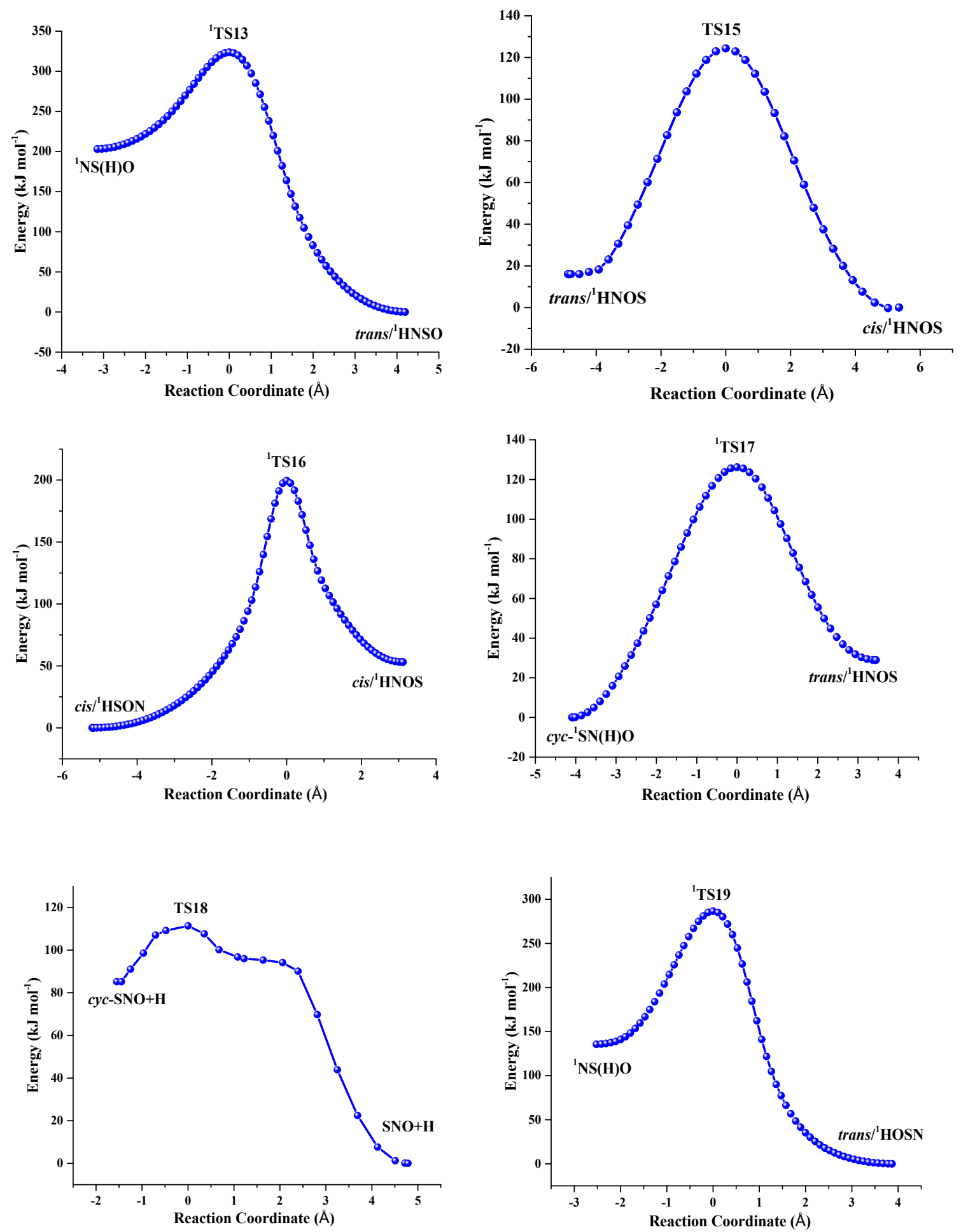

Figure S2. Calculated IRC for ${ }^{1} \mathrm{R}_{13},{ }^{1} \mathrm{R}_{15},{ }^{1} \mathrm{R}_{16},{ }^{1} \mathrm{R}_{17},{ }^{1} \mathrm{R}_{18}$, and ${ }^{1} \mathrm{R}_{19}$ reactions at $\mathrm{B} 3 \mathrm{LYP} / \mathrm{CBSB} 7$ level. 

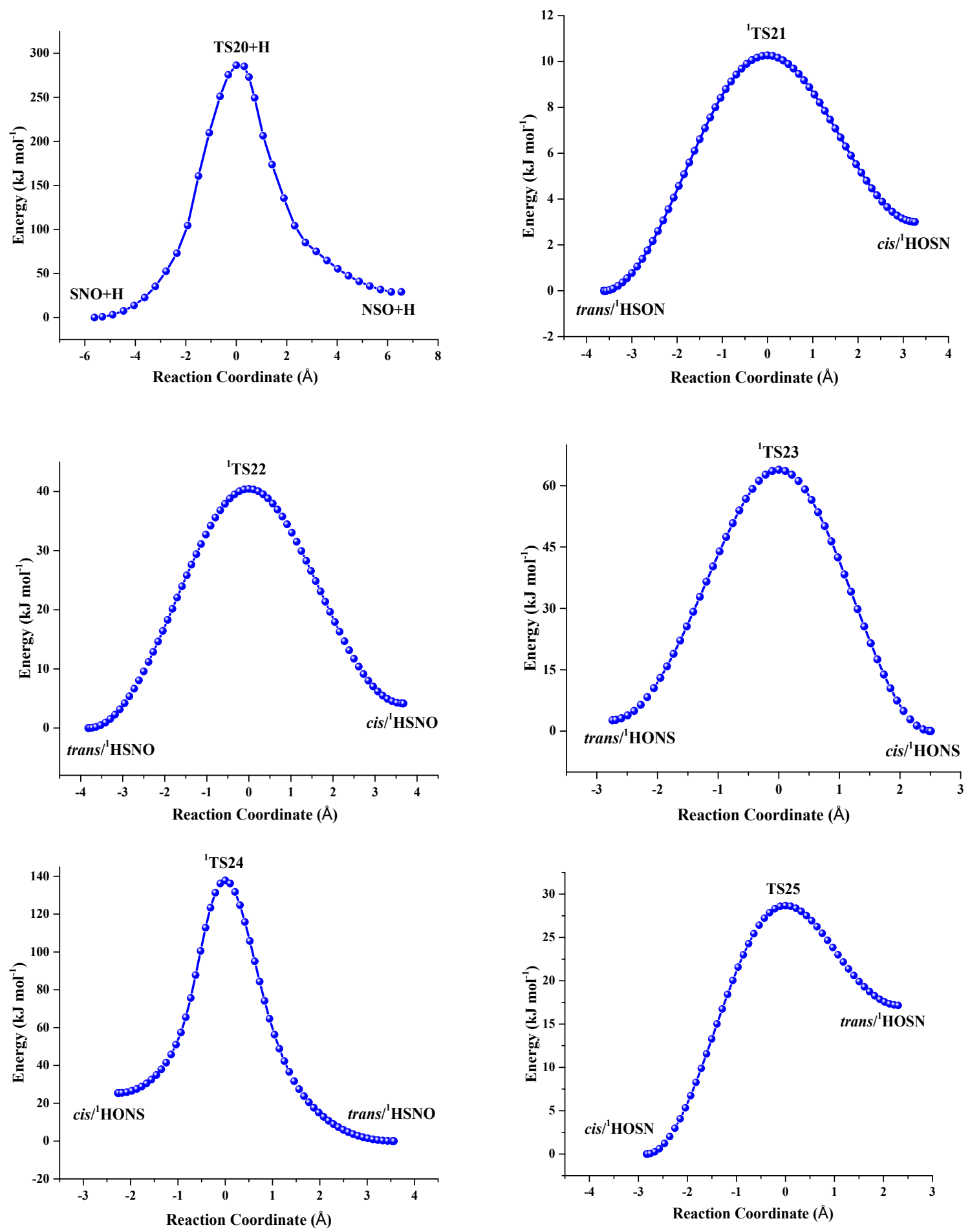

Figure S3. Calculated IRC for ${ }^{1} \mathrm{R}_{20},{ }^{1} \mathrm{R}_{21},{ }^{1} \mathrm{R}_{22},{ }^{1} \mathrm{R}_{23},{ }^{1} \mathrm{R}_{24}$ and ${ }^{1} \mathrm{R}_{25}$ reactions at B3LYP/CBSB7 level. 

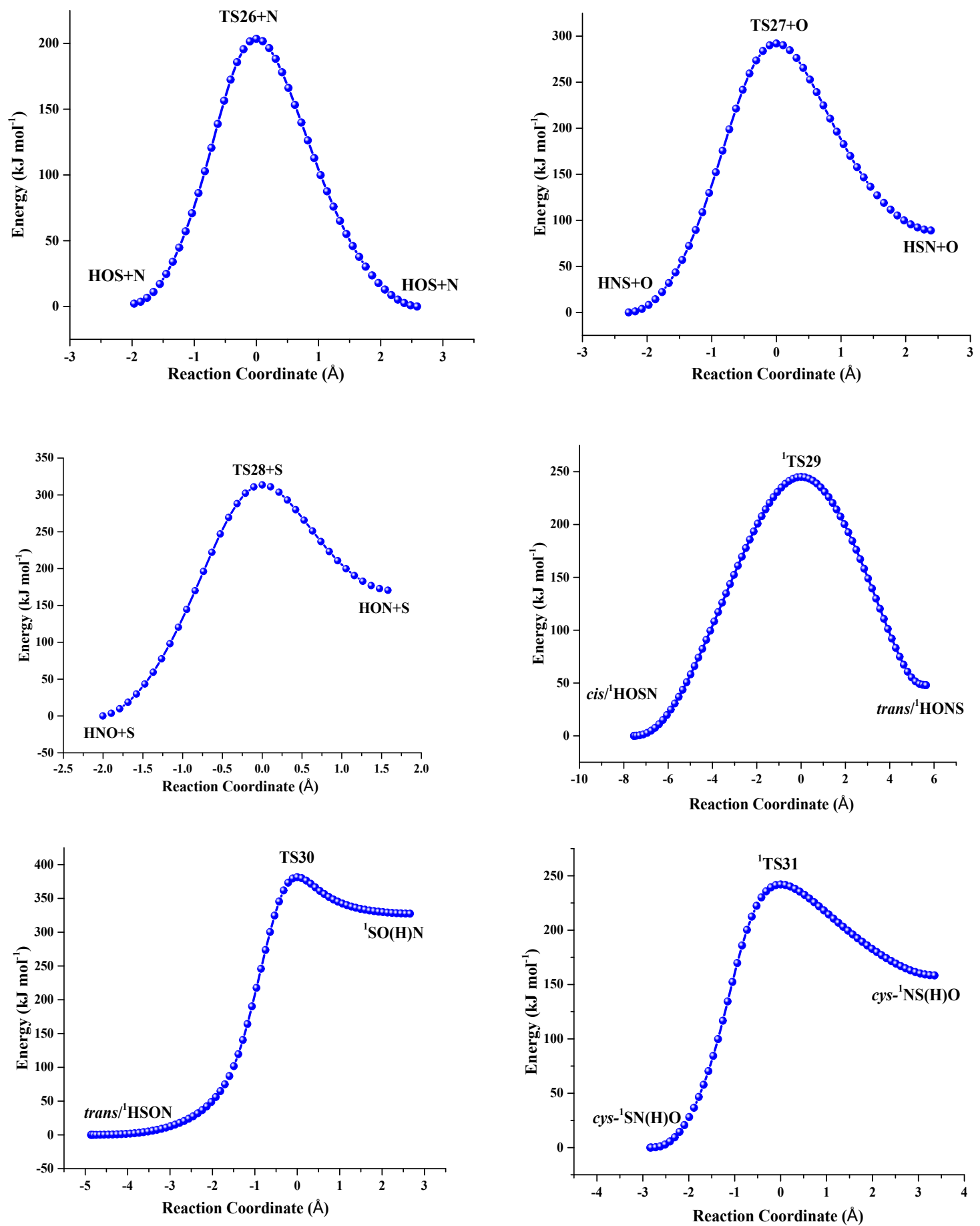

Figure S4. Calculated IRC for ${ }^{1} \mathrm{R}_{26},{ }^{1} \mathrm{R}_{27},{ }^{1} \mathrm{R}_{28},{ }^{1} \mathrm{R}_{29},{ }^{1} \mathrm{R}_{30}$ and ${ }^{1} \mathrm{R}_{31}$ reactions at $\mathrm{B} 3 \mathrm{LYP} / \mathrm{CBSB} 7$ level. 

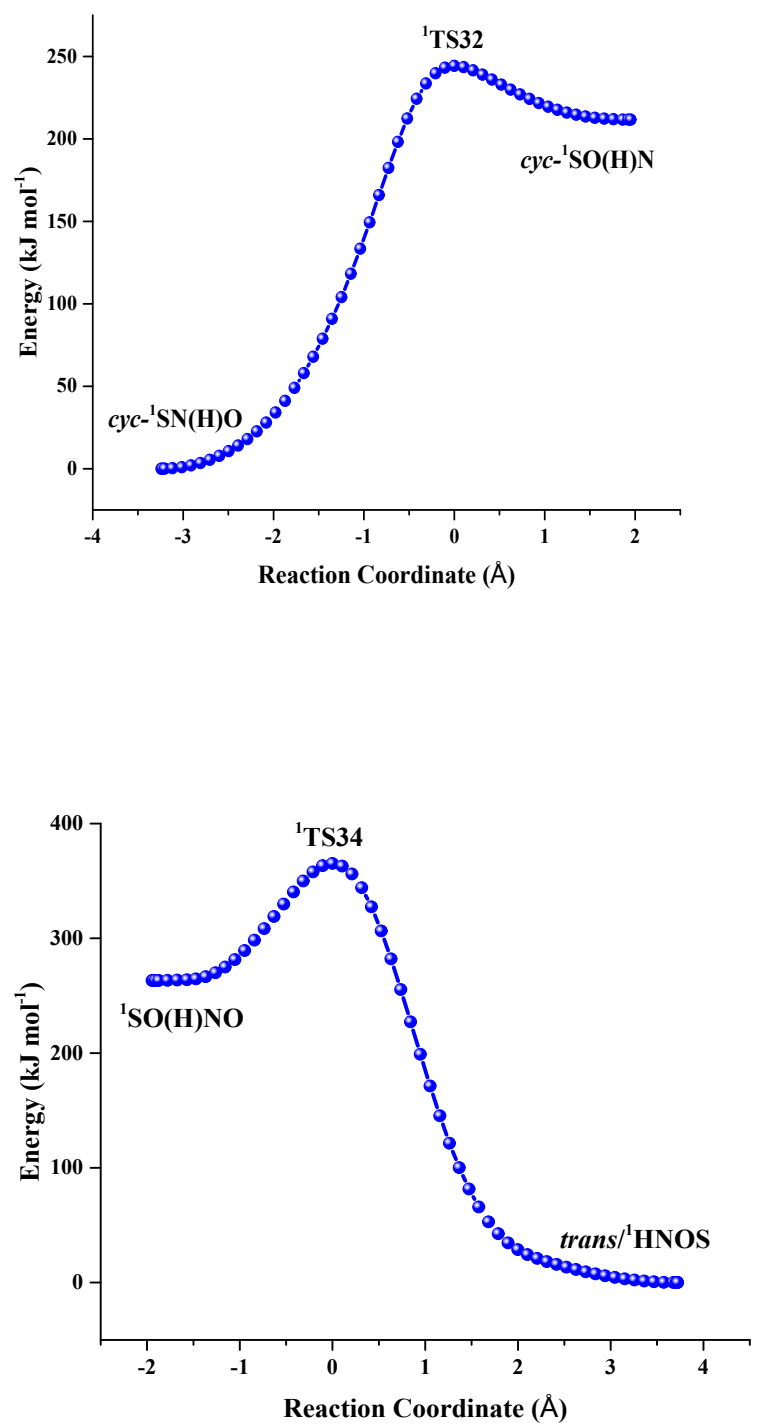

Figure S5. Calculated IRC for ${ }^{1} \mathrm{R}_{32}$ and ${ }^{1} \mathrm{R}_{34}$ reactions at $\mathrm{B} 3 \mathrm{LYP} / \mathrm{CBSB} 7$ level. 

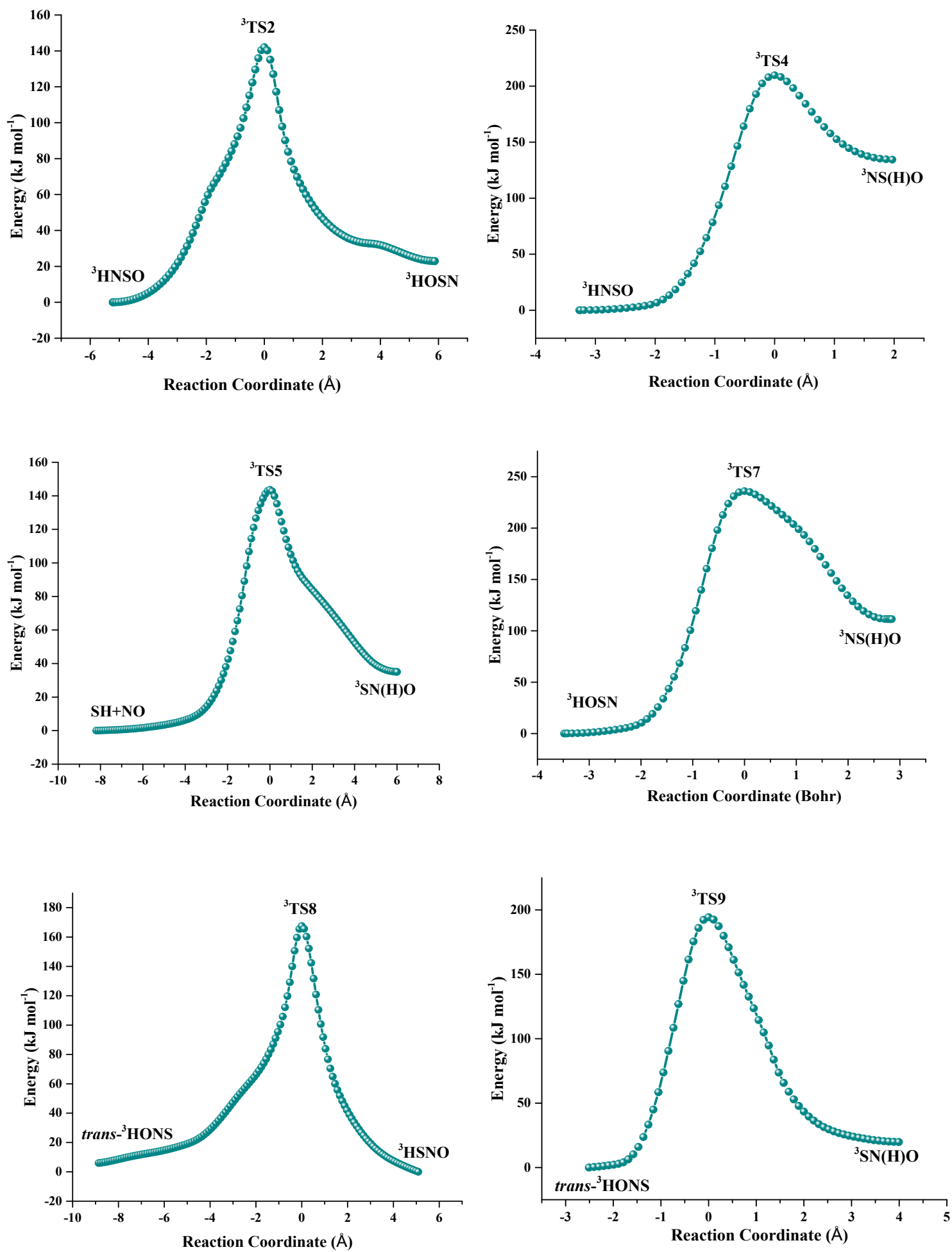

Figure S6. Calculated IRC for ${ }^{3} \mathrm{R}_{2},{ }^{3} \mathrm{R}_{4},{ }^{3} \mathrm{R}_{5},{ }^{3} \mathrm{R}_{7},{ }^{3} \mathrm{R}_{8}$ and ${ }^{3} \mathrm{R}_{9}$ reactions at B3LYP/CBSB7 level. 

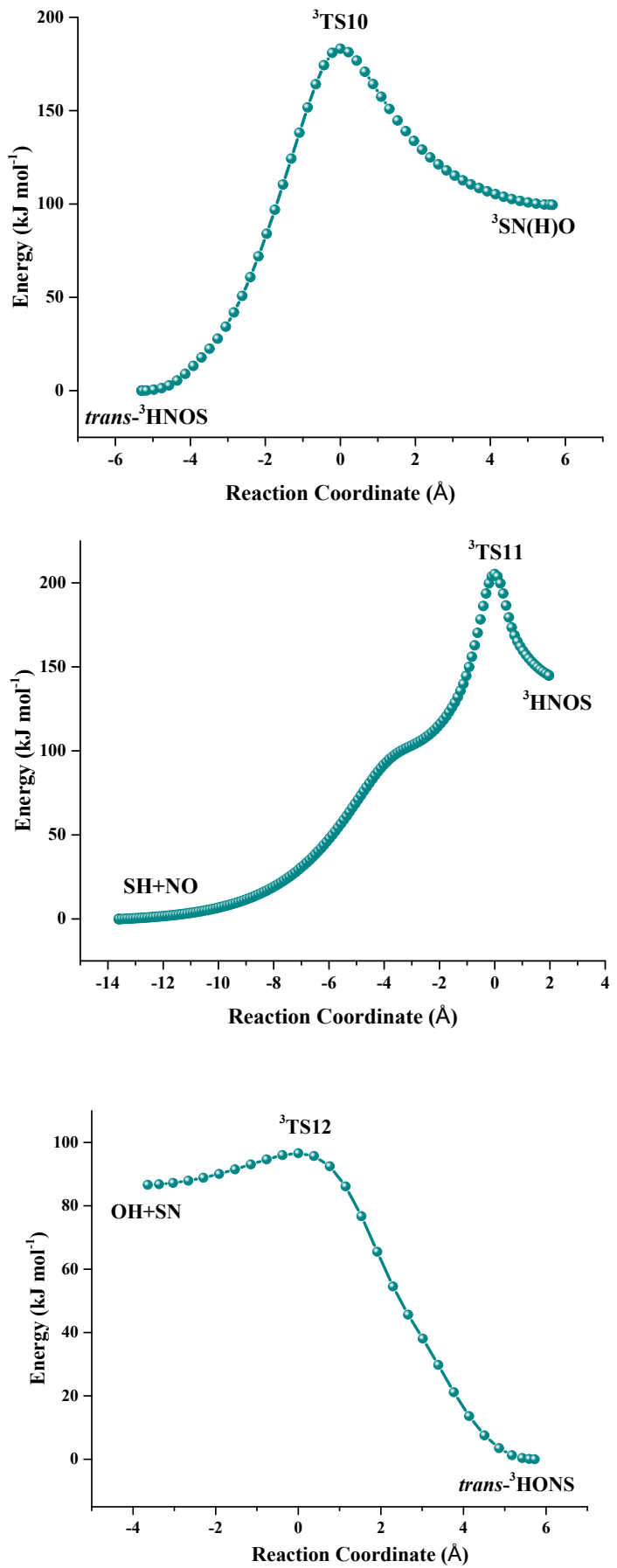

Figure S7. Calculated IRC for ${ }^{3} \mathrm{R}_{10},{ }^{3} \mathrm{R}_{11}$ and ${ }^{3} \mathrm{R}_{12}$ reactions at B3LYP/CBSB7 level. 
a)

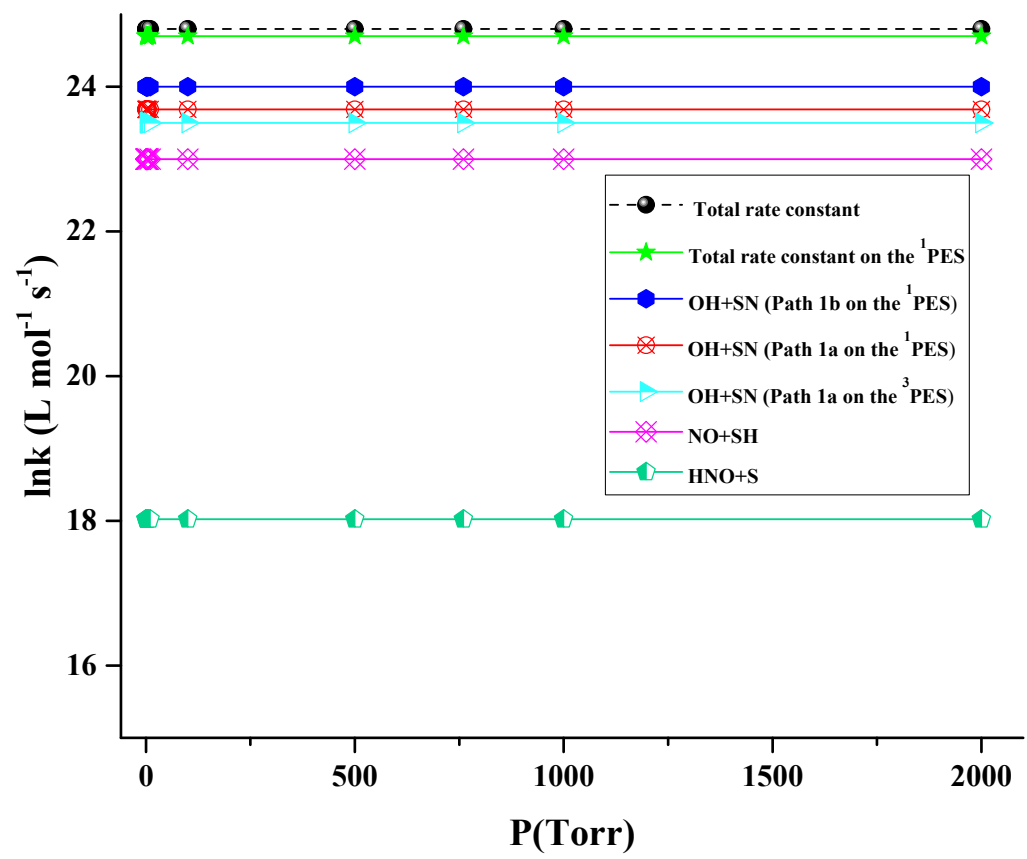

b)

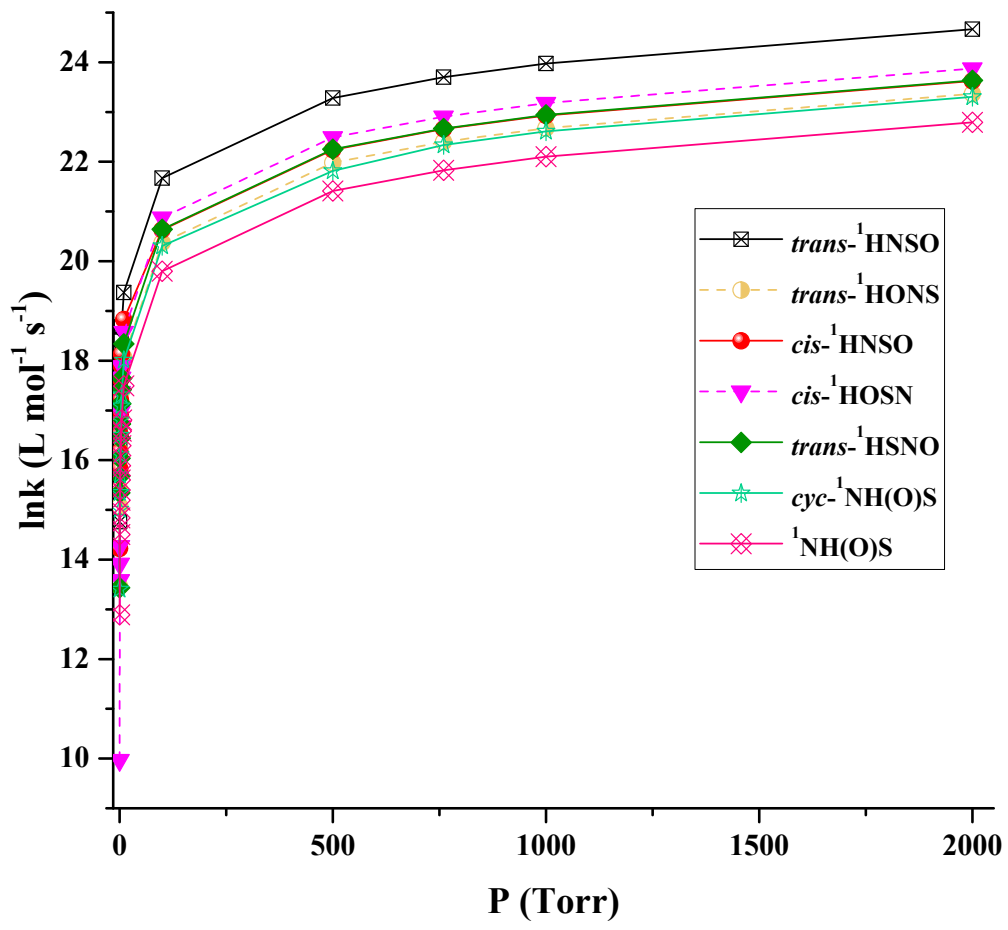

Figure S8. The Effect of pressure on a) the total rate constant and the rate of the formation of the main products on the ${ }^{1} \mathrm{PES}$ and ${ }^{3} \mathrm{PES}$, at $500 \mathrm{~K} \mathrm{~b}$ ) the rate of association, isomerization or decomposition of the energized intermediated on the ${ }^{1} \mathrm{PES}$, at $500 \mathrm{~K}$. 


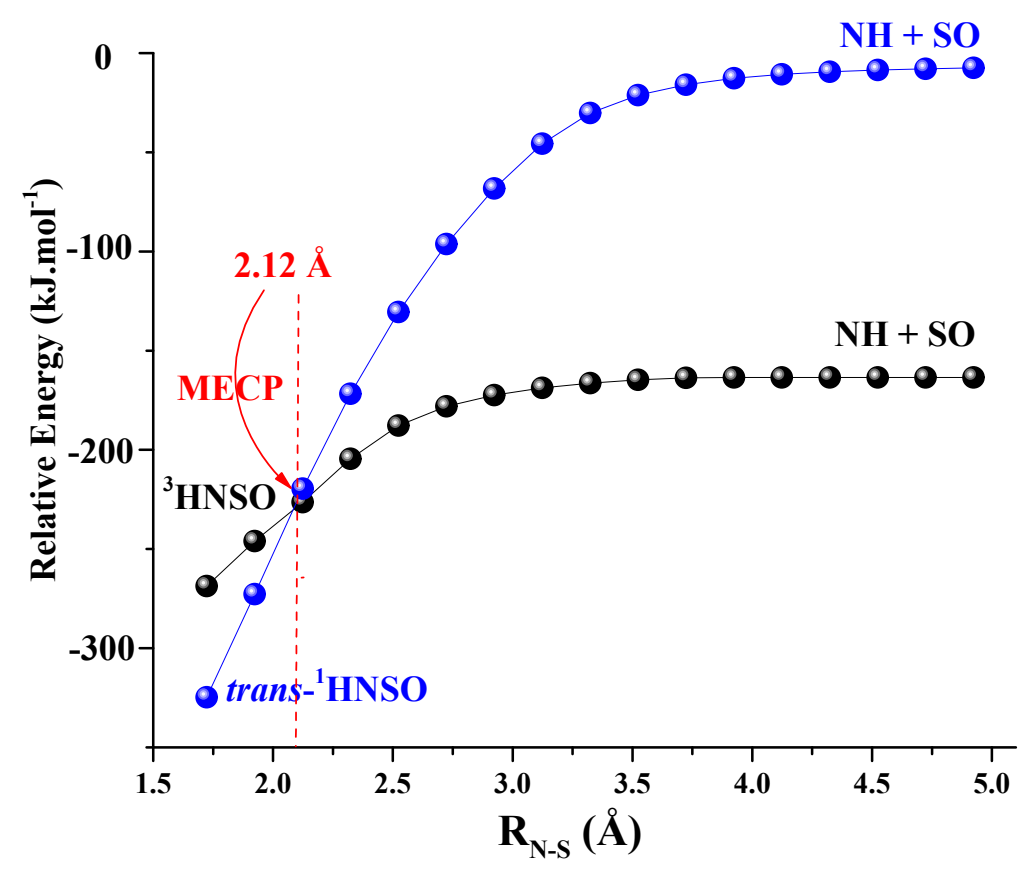

Figure S9. Minimal energy crossing point (MECP) for association reactions of the title reaction to form trans- ${ }^{-} \mathrm{HNSO}$ or ${ }^{3} \mathrm{HNSO}$ calculated at the CBS-QB3 level. 


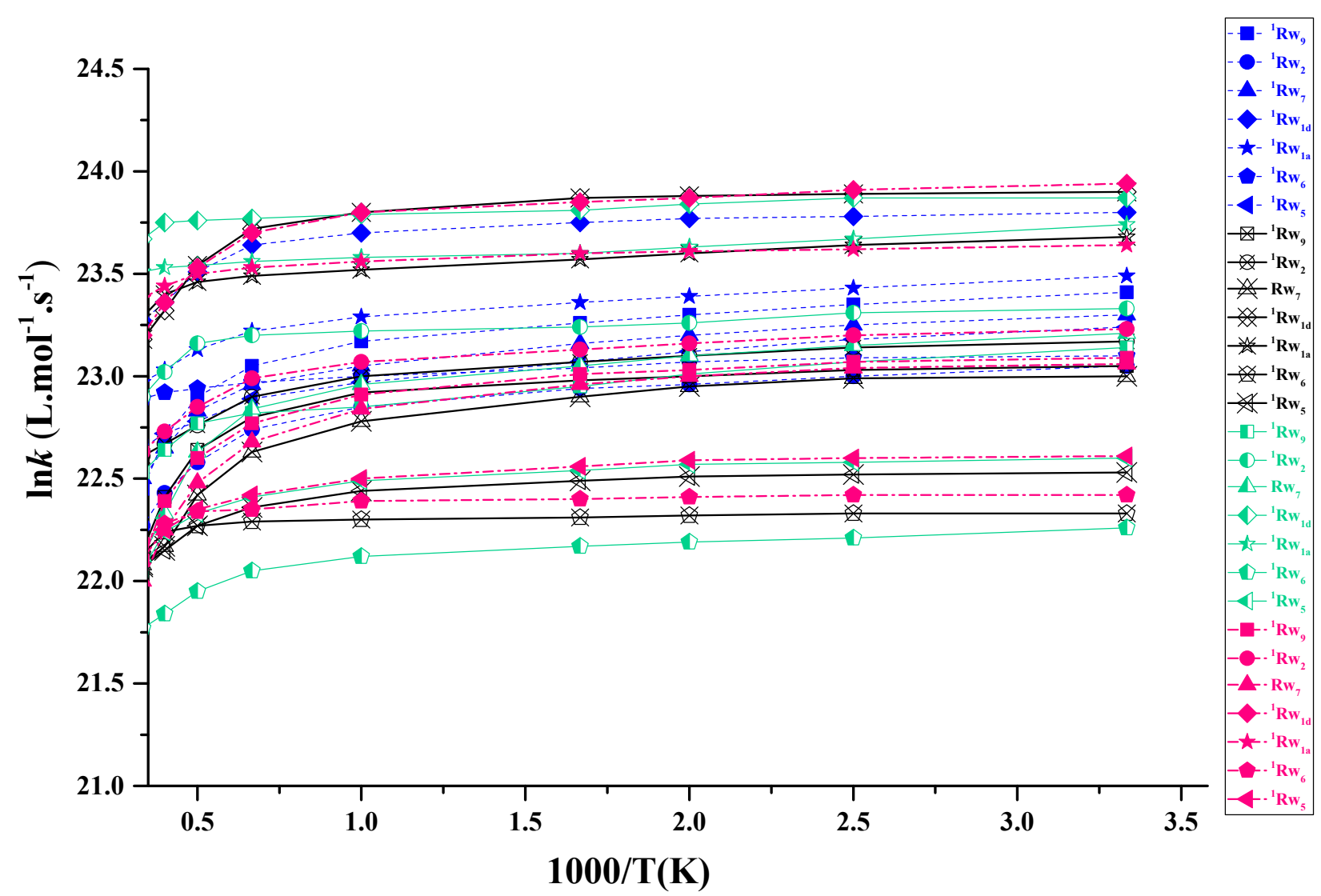

Figure S10. The Arrhenius plot of $\mathrm{k}_{\mathrm{w}}$ for assigned paths on the ${ }^{1} \mathrm{PES}$ calculated by the RRKMSSA method at 760 Torr and at different levels of theory. CASSCF (blue), CASPT2//CASSCF (black), CCSD(T)//B3LYP (green), and CASPT2//B3LYP(pink). 
Table S8. The corresponding rate expression in the $\mathrm{L} \mathrm{mol}^{-1} \mathrm{~s}^{-1}$ units from the energies calculated at the CCSD(T)//B3LYP, CASSCF, CASPT2//CASSCF, and CASPT2//B3LYP levels.

\section{CASSCF}

\section{CASPT2/CASSCF}

\begin{tabular}{|c|c|c|}
\hline${ }^{1} \mathbf{R w}_{\mathbf{9}}$ & $\mathrm{k}=1.73 \times 10^{12} \mathrm{~T}^{-0.69} \exp \left(-2.25 \mathrm{kJmol}^{-1} / \mathrm{T}\right)$ & $\mathrm{k}=2.72 \times 10^{12} \mathrm{~T}^{-0.78} \exp \left(-3.03 \mathrm{kJmol}^{-1} / \mathrm{T}\right)$ \\
\hline${ }^{1} \mathbf{R w}_{2}$ & $\mathrm{k}=5.80 \times 10^{11} \mathrm{~T}^{-0.58} \exp \left(-1.88 \mathrm{kJmol}^{-1} / \mathrm{T}\right)$ & $\mathrm{k}=1.82 \times 10^{11} \mathrm{~T}^{-0.41} \exp \left(-1.13 \mathrm{kJmol}^{-1} / \mathrm{T}\right)$ \\
\hline${ }^{1} \mathbf{R w}_{7}$ & $\mathrm{k}=5.55 \times 10^{11} \mathrm{~T}^{-0.55} \exp \left(-1.60 \mathrm{kJmol}^{-1} / \mathrm{T}\right)$ & $\mathrm{k}=2.47 \times 10^{12} \mathrm{~T}^{-0.79} \exp \left(-2.67 \mathrm{kJmol}^{-1} / \mathrm{T}\right)$ \\
\hline${ }^{1} \mathbf{R} \mathbf{w}_{1 \mathrm{~d}}$ & $\mathrm{k}=6.01 \times 10^{11} \mathrm{~T}^{-0.46} \exp \left(-1.74 \mathrm{kJmol}^{-1} / \mathrm{T}\right)$ & $\mathrm{k}=3.63 \times 10^{12} \mathrm{~T}^{-0.69} \exp \left(-2.79 \mathrm{kJmol}^{-1} / \mathrm{T}\right)$ \\
\hline${ }^{1} \mathbf{R} \mathbf{w}_{1 \mathbf{a}}$ & $\mathrm{k}=1.12 \times 10^{11} \mathrm{~T}^{-0.30} \exp \left(-0.62 \mathrm{kJmol}^{-1} / \mathrm{T}\right)$ & $\mathrm{k}=5.94 \times 10^{10} \mathrm{~T}^{-0.18} \exp \left(-0.28 \mathrm{kJmol}^{-1} / \mathrm{T}\right)$ \\
\hline${ }^{1} \mathbf{R w}_{6}$ & $\mathrm{k}=2.04 \times 10^{10} \mathrm{~T}^{-0.11} \exp \left(-0.10 \mathrm{kJmol}^{-1} / \mathrm{T}\right)$ & $\mathrm{k}=1.45 \times 10^{10} \mathrm{~T}^{-0.15} \exp \left(-0.60 \mathrm{kJmol}^{-1} / \mathrm{T}\right)$ \\
\hline${ }^{1} \mathbf{R w}_{5}$ & $\mathrm{k}=7.44 \times 10^{10} \mathrm{~T}^{-0.29} \exp \left(-0.31 \mathrm{kJmol}^{-1} / \mathrm{T}\right)$ & $\mathrm{k}=1.04 \times 10^{11} \mathrm{~T}^{-0.40} \exp \left(-1.44 \mathrm{kJmol}^{-1} / \mathrm{T}\right)$ \\
\hline & CCSD(T)//B3LYP & CASPT2/B3LYP \\
\hline${ }^{1} \mathbf{R w}_{\mathbf{9}}$ & $\mathrm{k}=5.66 \times 10^{10} \mathrm{~T}^{-0.27} \exp \left(-0.26 \mathrm{kJmol}^{-1} / \mathrm{T}\right)$ & $\mathrm{k}=3.63 \times 10^{12} \mathrm{~T}^{-0.82} \exp \left(-3.04 \mathrm{kJmol}^{-1} / \mathrm{T}\right)$ \\
\hline${ }^{1} \mathbf{R w}_{2}$ & $\mathrm{k}=9.57 \times 10^{10} \mathrm{~T}^{-0.28} \exp \left(-0.93 \mathrm{kJmol}^{-1} / \mathrm{T}\right)$ & $\mathrm{k}=2.67 \times 10^{11} \mathrm{~T}^{-0.45} \exp \left(-1.39 \mathrm{kJmol}^{-1} / \mathrm{T}\right)$ \\
\hline${ }^{1} \mathbf{R w}_{7}$ & $\mathrm{k}=4.68 \times 10^{12} \mathrm{~T}^{-0.85} \exp \left(-2.94 \mathrm{kJmol}^{-1} / \mathrm{T}\right)$ & $\mathrm{k}=5.06 \times 10^{12} \mathrm{~T}^{-0.88} \exp \left(-3.11 \mathrm{kJmol}^{-1} / \mathrm{T}\right)$ \\
\hline${ }^{1} \mathbf{R} \mathbf{w}_{1 \mathrm{~d}}$ & $\mathrm{k}=3.98 \times 10^{10} \mathrm{~T}^{-0.01} \exp \left(-0.10 \mathrm{kJmol}^{-1} / \mathrm{T}\right)$ & $\mathrm{k}=1.82 \times 10^{12} \mathrm{~T}^{-0.61} \exp \left(-2.19 \mathrm{kJmol}^{-1} / \mathrm{T}\right)$ \\
\hline${ }^{1} \mathbf{R w}_{1 \mathbf{a}}$ & $\mathrm{k}=1.59 \times 10^{10} \exp \left(-0.58 \mathrm{kJmol}^{-1} / \mathrm{T}\right)$ & $\mathrm{k}=6.22 \times 10^{10} \mathrm{~T}^{-0.18} \exp \left(-0.53 \mathrm{kJmol}^{-1 / T}\right)$ \\
\hline${ }^{1} \mathbf{R w}_{6}$ & $\mathrm{k}=2.60 \times 10^{10} \mathrm{~T}^{-0.29} \exp \left(-0.24 \mathrm{kJmol}^{-1} / \mathrm{T}\right)$ & $\mathrm{k}=2.09 \times 10^{10} \mathrm{~T}^{-0.19} \exp \left(-0.73 \mathrm{kJmol}^{-1} / \mathrm{T}\right)$ \\
\hline${ }^{1} \mathbf{R} \mathbf{w}_{5}$ & $\mathrm{k}=1.24 \times 10^{11} \mathrm{~T}^{-0.42} \exp \left(-1.48 \mathrm{kJmol}^{-1} / \mathrm{T}\right)$ & $\mathrm{k}=1.09 \times 10^{11} \mathrm{~T}^{-0.40} \exp \left(-1.38 \mathrm{kJmol}^{-1} / \mathrm{T}\right)$ \\
\hline
\end{tabular}


Table S9. Computed $\mathrm{T} 1$ values for various species on the ${ }^{1} \mathrm{PES}$ at $\operatorname{CCSD}(\mathrm{T}) /$ aug-cc$\mathrm{pVTZ//B3LYP/CBSB7}$ level of calculations.

\begin{tabular}{|c|c|}
\hline $\begin{array}{c}\text { Stationary } \\
\text { Point }\end{array}$ & T1 \\
\hline cis-1$^{1} \mathrm{HNOS}$ & 0.060 \\
\hline cis ${ }^{1}$ HNSO & 0.021 \\
\hline cis ${ }^{1} \mathrm{HONS}$ & 0.022 \\
\hline cis ${ }^{-1} \mathrm{HOSN}$ & 0.032 \\
\hline cis - ${ }^{1}$ HSNO & 0.022 \\
\hline cis ${ }^{-1} \mathrm{HSON}$ & 0.100 \\
\hline trans- ${ }^{1}$ HNOS & 0.046 \\
\hline trans $-{ }^{1} \mathrm{HNSO}$ & 0.039 \\
\hline trans $-{ }^{1} \mathrm{HONS}$ & 0.028 \\
\hline trans $-{ }^{1} \mathrm{HOSN}$ & 0.034 \\
\hline trans $-{ }^{1} \mathrm{HSNO}$ & 0.025 \\
\hline trans $-{ }^{1} \mathrm{HSON}$ & 0.089 \\
\hline${ }^{1} \mathrm{~N}(\mathrm{H}) \mathrm{SO}$ & 0.027 \\
\hline${ }^{1} \mathrm{~S}(\mathrm{H}) \mathrm{NO}$ & 0.018 \\
\hline${ }^{1} \mathrm{O}(\mathrm{H}) \mathrm{SN}$ & 0.054 \\
\hline$c y c-1 \mathbf{N}(\mathbf{H}) \mathrm{SO}$ & 0.017 \\
\hline$c y c-{ }^{1} \mathrm{~S}(\mathrm{H}) \mathrm{NO}$ & 0.022 \\
\hline$c y c-1 \mathrm{O}(\mathrm{H}) \mathrm{SN}$ & 0.024 \\
\hline${ }^{1} \mathrm{TS} 1$ & 0.034 \\
\hline${ }^{1}$ TS2 & 0.021 \\
\hline${ }^{1}$ TS3 & 0.030 \\
\hline${ }^{1}$ TS4 & 0.019 \\
\hline${ }^{1}$ TS5 & 0.071 \\
\hline${ }^{1}$ TS6 & 0.021 \\
\hline${ }^{1}$ TS7 & 0.028 \\
\hline${ }^{1}$ TS8 & 0.115 \\
\hline${ }^{1}$ TS9 & 0.044 \\
\hline${ }^{1}$ TS10 & 0.051 \\
\hline${ }^{1}$ TS11 & 0.051 \\
\hline${ }^{1}$ TS12 & 0.078 \\
\hline${ }^{1}$ TS13 & 0.029 \\
\hline${ }^{1}$ TS14 & 0.029 \\
\hline${ }^{1}$ TS15 & 0.082 \\
\hline${ }^{1}$ TS16 & 0.072 \\
\hline${ }^{1}$ TS17 & 0.039 \\
\hline${ }^{1}$ TS18 & 0.063 \\
\hline${ }^{1}$ TS19 & 0.021 \\
\hline${ }^{1}$ TS20 & 0.046 \\
\hline${ }^{1}$ TS21 & 0.028 \\
\hline${ }^{1}$ TS22 & 0.027 \\
\hline${ }^{1}$ TS23 & 0.096 \\
\hline
\end{tabular}




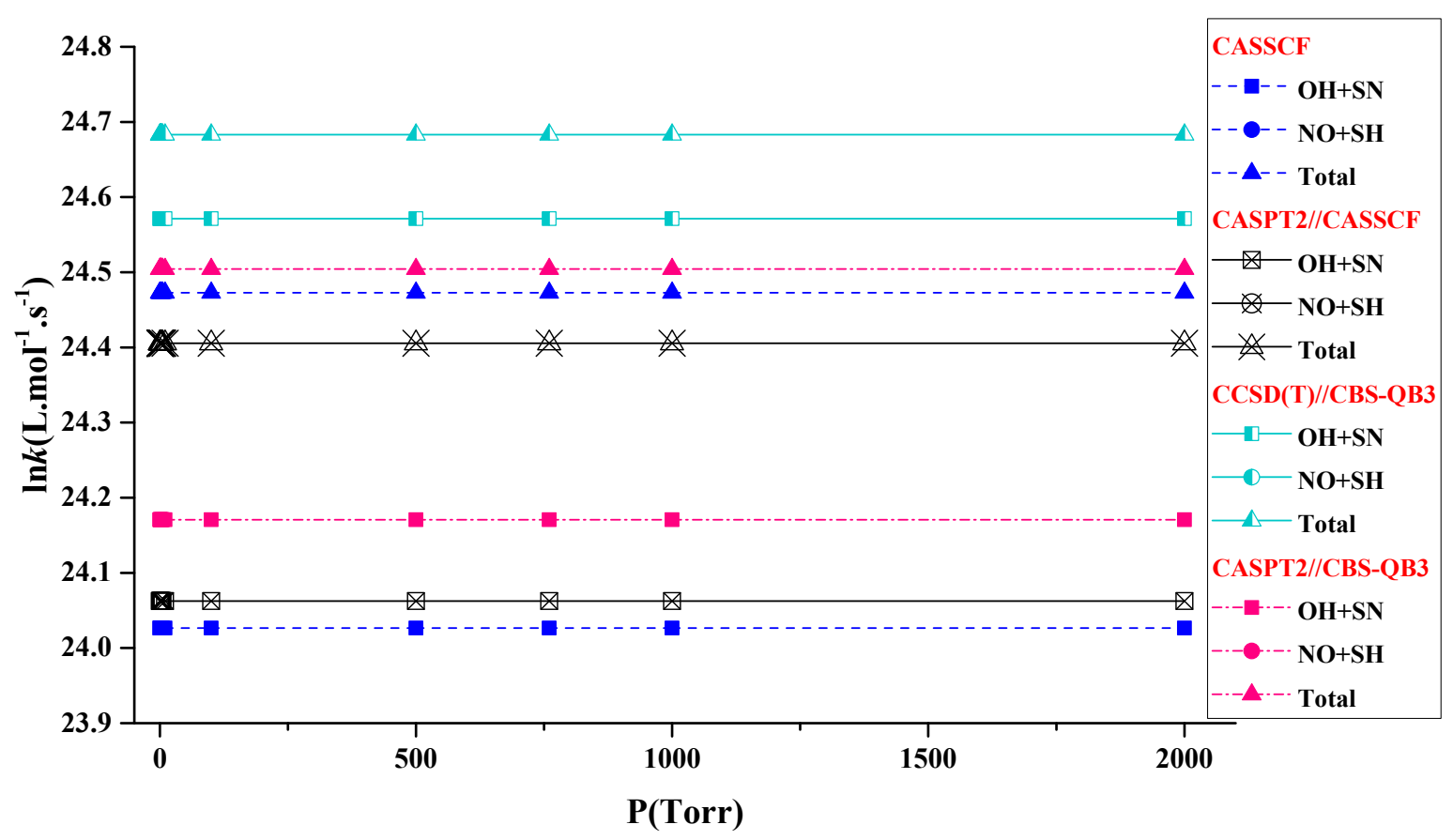

Figure S11. The Effect of pressure on the total rate constants and and the rate of the major products formation of the title reaction on the singlet surface, at $500 \mathrm{~K}$, at different levels of theory.

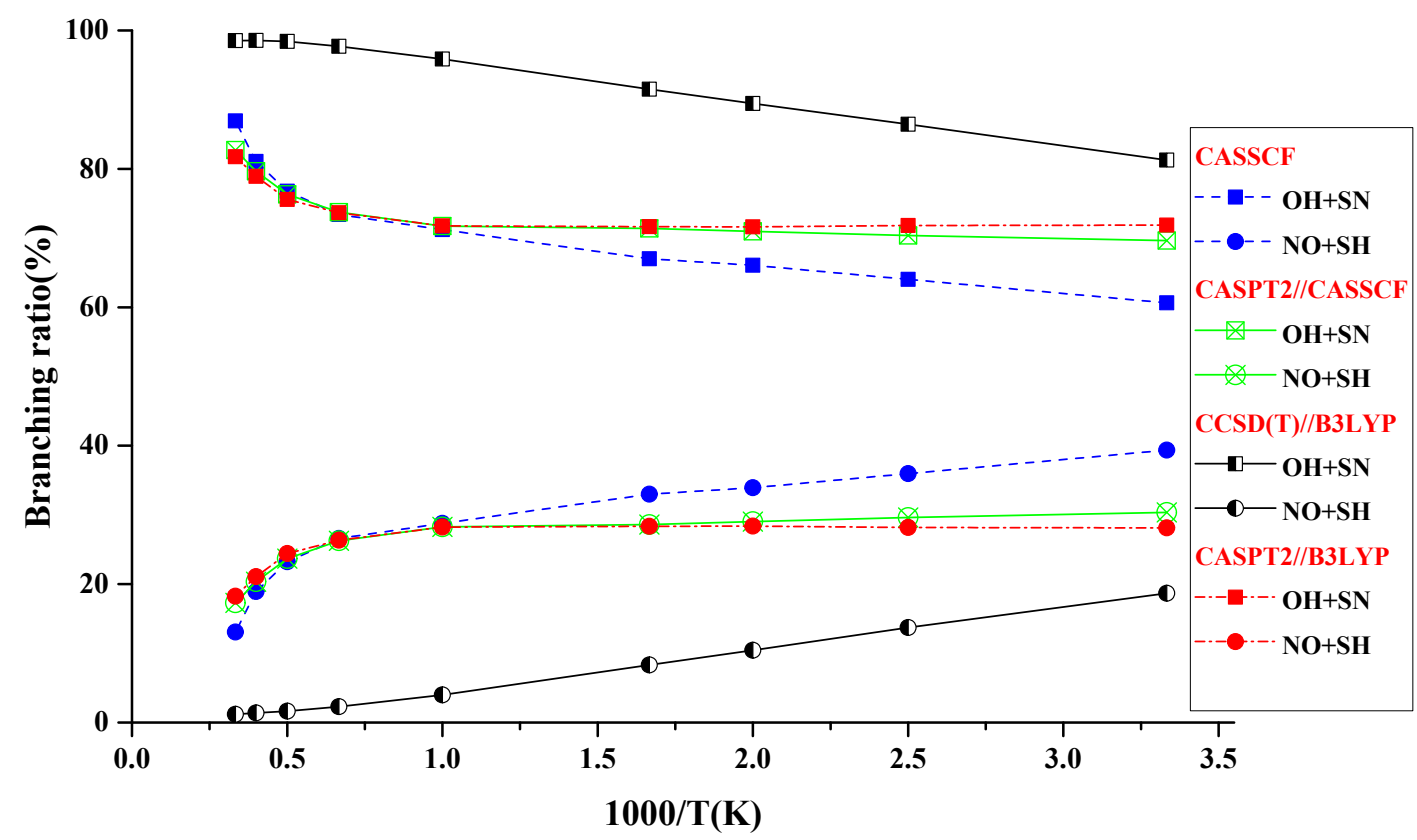

Figure S12. The branching ratios for the major products of $\mathrm{NH}\left(\mathrm{X}^{3} \Sigma^{-}\right)+\mathrm{SO}\left(\mathrm{X}^{3} \Sigma^{-}\right)$reaction on the singlet surface at 760 Torr, at different levels of theory. 